\title{
FACTORES QUE AFECTAN LA PRODUCCION DE SEMILLAS EN PSEUDOTSUGA MACROLEPIS FLOUS EN EL ESTADO DE HIDALGO, MEXICO
}

\author{
Fernando Zavala Chavez \\ Laboratorio de Ecología Forestal \\ División de Ciencias Forestales \\ Universidad Autónoma Chapingo \\ Apartado Postal 084 \\ 56230 Chapingo, México \\ Y \\ Jose Tulio Mendez Montiel \\ Laboratorio de Entomología Forestal \\ División de Ciencias Forestales \\ Universidad Autónoma Chapingo \\ 56230 Chapingo, México
}

\begin{abstract}
RESUMEN
El propósito de este trabajo fue investigar el efecto de la polinización y la incidencia de insectos en la calidad y cantidad de las semillas de Pseudotsuga macrolepis, disponibles para su regeneración en la porción occidental de la Sierra de Pachuca, Hgo. La polinización y la incidencia de insectos fueron controladas experimentalmente. Los resultados evidencian que algunas semillas pudieron haber sido desarrolladas por partenogénesis y que la polinización ineficiente y el ataque de insectos pueden disminuir la cantidad de semillas potenciales y producidas. Debido a que prácticamente no existen semillas para la regeneración natural de $P$. macrolepis, se plantea la necesidad de una repoblación artificial de dicha especie.
\end{abstract}

\begin{abstract}
The purpose of this work was to investigate the effect of pollination and insects incidence in both quality and quantity of seeds of Pseudotsuga macrolepis available for its natural regeneration in the Western portion of Sierra de Pachuca, state of Hidalgo. Pollination and insects incidence were controlled experimentally. Results appear to indicate that some seeds were developed through parthenogenesis. In addition, ineffective pollination and attack of insects can decrease the amount of potential and produced seeds. Because there are practically no seeds for natural regeneration, we conclude that an artificial repopulation of $P$. macrolepis is necessary.
\end{abstract}




\section{INTRODUCCION}

Las especies de Pseudotsuga de México han sido poco estudiadas y a menudo hasta relegadas, principalmente en los aspectos relacionados con su biología. En países como Estados Unidos y Canadá, Pseudotsuga es uno de los géneros de coníferas económicamente más importantes y es el más utilizado como árbol de navidad; en México suele importarse con este propósito. Es urgente hacer algo para rescatar las áreas de bosque de Pseudotsuga que han disminuido a ritmos acelerados en nuestro país, principalmente por la tala indiscriminada y la apertura de nuevas tierras para cultivos agrícolas (papa, cebada y avena, básicamente). Esto ha estado ocurriendo en Hidalgo, Tlaxcala y Veracruz, entidades en las que se localizan los límites meridionales de la distribución de Pseudotsuga en América.

El uso de las especies nativas para los fines de reforestación es deseable en el país, pero para ello se requiere semilla de buena calidad. Sin embargo, su producción es afectada por diversos factores, tales como las fluctuaciones climáticas bruscas, la polinización y la fertilización inadecuadas, la deficiencia de nutrientes en el suelo, así como la depredación de conos y semillas por insectos y otros animales.

En la porción occidental de la Sierra de Pachuca, Hgo., observaciones preliminares mostraron serios problemas en la producción de semillas en $P$. macrolepis. Aunque no se conocen las causas, se han encontrado semillas vanas en altas cantidades. La distribución de esta especie en la zona se restringe a pequeños manchones aislados con densidades bajas y su estrategia regenerativa parece ser exclusivamente por semilla. De continuar la disminución del número de individuos de $P$. macrolepis y el disturbio de su hábitat, esta especie puede estar en peligro de desaparecer del área.

Según Owens (1986), existen factores endógenos y exógenos que pueden afectar el desarrollo de los óvulos, semillas y conos, pero aún no están bien entendidos. Por ejemplo, el aborto de conillos femeninos de pinos después de la polinización es común y se debe principalmente a las bajas temperaturas (Hutchinson y Bramlett, 1964). Según Sarvas (1962), citado por Owens (1986), cuando más de $20 \%$ de los óvulos potencialmente fértiles de Pinus sylvestris L. abortaron, se observó deficiencia en la polinización. También se ha registrado pérdida de conillos después de la fertilización, pero tal fenómeno no es común (Owens, 1986). Algunos autores consignan que la mortalidad de conos de pinos está relacionada con un déficit de humedad (Rehfeldt et al., 1971).

El período de germinación del polen varía en los conos femeninos y durante el año entre las diferentes especies de pináceas. Aunque todavía no se conoce qué la estimula, se cree que la autopolinización, humedad y temperatura pueden afectar su velocidad; Owens (1986) propone que dichos factores podrían reducir el tamaño de las semillas si la germinación del polen se adelantara, o bien podrían disminuir el crecimiento del tubo polínico si esta última se retrasara.

La carencia de óvulos en las regiones fértiles del cono se puede deber a que éstos hayan abortado o a un desarrollo incompleto. Cualquiera que sea la causa, su ausencia reduce significativamente el número de semillas viables o llenas, o aumenta el número de las vanas (Owens, 1986). El carácter vano ha sido adjudicado a otros factores, especialmente los insectos; por ejemplo, Leptoglossus occidentalis (chinche, Hemiptera) es la causa principal de la presencia de semillas vacías en Pinus cembroides Zucc. en 
México, así como en Pseudotsuga menziesii en Estados Unidos de América y Canadá. Dicho insecto se encuentra ampliamente distribuido en México y afecta a más de 17 especies de Pinus (Cibrián et al., 1986).

Una hipótesis aceptada es la que considera a la autopolinización como causa principal del desarrollo de semillas vanas, ya que provoca la manifestación de genes letales. Tal fenómeno produce la muerte de los embriones, por lo cual las semillas de los conos maduros están vacías. Sin embargo, la presencia de éstas también puede tener una explicación adaptativa, pues se considera como una defensa contra su depredación (Willson, 1983).

El objetivo de este trabajo fue investigar el efecto de la polinización y de la incidencia de insectos en la calidad y cantidad de semillas disponibles para la regeneración de $P$. macrolepis en una población natural de la porción occidental de la Sierra de Pachuca, en el Ejido El Cerezo, municipio de Pachuca, Hidalgo.

\section{METODOLOGIA}

Area de estudio

El trabajo se realizó en terrenos aledaños a la Presa Jaramillo, pertenecientes al Ejido El Cerezo, municipio de Pachuca, Hgo., al norte de la ciudad de Pachuca y a unos $24 \mathrm{~km}$ de ésta por la carretera a Mineral El Chico (Fig. 1). El área forma parte de la porción oeste de la Sierra de Pachuca y, según Medina y Rzedowski (1981), se caracteriza por su flora rica y diversa. El sustrato geológico está formado por rocas volcánicas, variando de riolíticas a basálticas, con predominancia de dacitas y andesitas. El terreno es accidentado con pendientes abruptas, aunque hay algunas áreas con escaso relieve. La altitud varía de 2700 hasta cerca de $3000 \mathrm{~m}$. Los vientos dominantes son del norte y noreste; el clima es relativamente fresco del tipo $\mathrm{C}(\mathrm{m}) \mathrm{b}(\mathrm{i}) \mathrm{g}$, que corresponde al templado húmedo con lluvias principalmente en verano (García, 1973); las temperaturas medias anuales varían de 10 a $14^{\circ} \mathrm{C}$ y las mínimas extremas entre -6 y $9{ }^{\circ} \mathrm{C}$; la precipitación total fluctúa entre 600 y $1500 \mathrm{~mm}$ en promedio anual; las neblinas y el rocío son frecuentes y en ocasiones se observa escarcha (Medina y Rzedowski, 1981). La vegetación es de bosque de Abies religiosa con Pseudotsuga, Arbutus, Quercus y Pinus, bosque de Quercus con Pseudotsuga y Pinus y bosque de Quercus-Pseudotsuga. Actualmente el área se encuentra muy alterada.

\section{Métodos}

Se eligieron seis árboles con diámetro a la altura del pecho de 25 a $35 \mathrm{~cm}$, con evidencias de producción de conos en años anteriores, buena ramificación y separados entre sí por lo menos $100 \mathrm{~m}$. Los árboles fueron marcados con una banda de pintura en el fuste. En 5 de ellos se eligieron 8 ramas de unos $30 \mathrm{~cm}$ de longitud y ubicadas en el tercio superior de la copa. Cada una de ellas fue utilizada como unidad experimental; el número de yemas femeninas o el de conillos en plena emergencia se registró en todas las ramas. Para cada árbol se aplicaron 8 tratamientos, de tal manera que cada uno fue 
una repetición del experimento. La asignación de los tratamientos fue aleatoria y estos fueron los siguientes:

Tratamiento

$T_{1}$
$T_{2}$
$T_{3}$
$T_{4}$
$T_{5}$
$T_{6}$
$T_{7}$
$T_{8}$

\section{Descripción}

\author{
Sin polinizar y sin protección \\ Autopolinización y sin protección \\ Polinización libre y sin protección \\ Polinización controlada y sin protección \\ Sin polinizar y con protección \\ Autopolinización y con protección \\ Polinización libre y con protección \\ Polinización controlada y con protección
}

Los tratamientos sin polinizar ( $\mathrm{T}_{1}$ y $\left.\mathrm{T}_{5}\right)$ consistieron en cubrir las ramas con bolsas de estraza durante el período de floración, habiendo eliminado previamente los conillos masculinos de la rama. En los que incluyeron protección ( $\left.T_{5}-T_{8}\right)$ se cubrieron las ramas con una malla de organza durante el desarrollo de los conos; para los de autopolinización ( $T_{2}$ y $\mathrm{T}_{6}$ ) se dejaron de 20-30 conillos masculinos de la misma rama y se cubrieron con una bolsa de estraza; en los de polinización libre ( $T_{3}$ y $\left.T_{7}\right)$ se dejaron las ramas sin tapar durante el período de floración; en los de polinización controlada ( $T_{4}$ y T8) se rociaron artificialmente con polen escogido y se cubrieron las ramas con bolsas de estraza. El polen usado en los tratamientos de polinización controlada provino de un sexto árbol, seleccionado para tal fin. Después de concluidos los tratamientos, se hicieron cinco evaluaciones, aproximadamente cada mes. En las primeras tres se registraron los conillos sobrevivientes, la cuarta fue de inspección y en la quinta se cosecharon los conos maduros, mismos que se estudiaron mediante la técnica del análisis de cono, de acuerdo con Bramlett et al. (1977). Los datos de supervivencia de conillos (o conos) y las variables derivadas del examen de conos se analizaron siguiendo el diseño de bloques al azar, prueba de comparaciones múltiples con el método de contrastes ortogonales, además de coeficientes de correlación (Steel y Torrie, 1985).

\section{RESULTADOS}

Los tratamientos (Cuadro 1) mostraron diferencias estadísticamente significativas en el número promedio de semillas llenas (NPSLL, $\mathrm{P}=.03$ ), de las abortadas (NPSA, $\mathrm{P}=.02$ ) y de las desarrolladas (NPSD, $\mathrm{P}=.04$ ) entre conos polinizados y no polinizados. Los últimos produjeron un promedio de 17.9 semillas vanas de cada 24.9 desarrolladas por cono y sólo 6.7 llenas. El número de las vanas o vacías (NPSV) fue ligeramente mayor en conos no polinizados que en los polinizados, pero no hubo diferencias significativas; entre conos con polinización controlada y libre las variables tampoco mostraron diferencias estadísticamente significativas. 


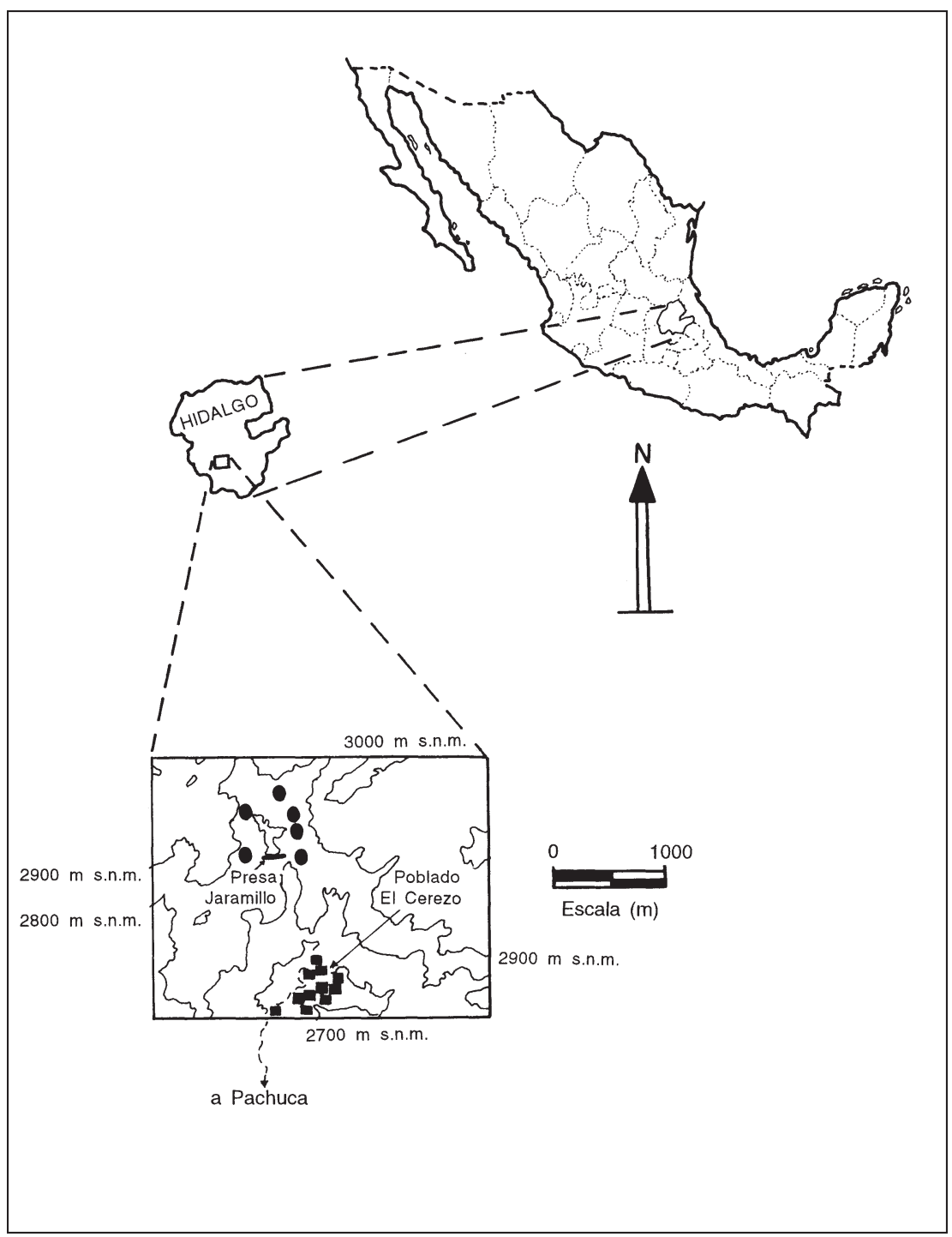

Fig. 1. Localización geográfica del área de estudio. Los óvalos negros representan la ubicación de los árboles estudiados. Basado en Anónimo, 1982.

De acuerdo con la cantidad de escamas fértiles (NPEF) de conos polinizados y no polinizados, el número promedio de semillas potenciales (NPSP) por cono fue de 51.5 (Cuadro 1). De ellas, $41 \%$ abortaron y $59 \%$ (30.5 semillas) lograron su desarrollo; de 
estas últimas $33 \%$ (10 semillas) resultaron vanas y sólo $25 \%$ quedaron disponibles para la regeneración; esto representa unas 8 semillas por cono.

$50 \%$ de los conos no polinizados carecieron de semillas llenas, $28.6 \%$ presentaron entre 1 y 10 y $21.4 \%$ entre 21 y 30 . Por otra parte, sólo $9.4 \%$ de los conos polinizados no presentaron semillas llenas, habiendo mostrado una mayor distribución de frecuencias en este carácter que en los conos no polinizados (Fig. 2).

Cuadro 1. Comparación entre el número de escamas y de semillas en conos polinizados y no polinizados de Pseudotsuga macrolepis de Hidalgo.

\begin{tabular}{lcrcc}
\hline Variable $^{*}$ & $\begin{array}{c}\text { Conos polinizados } \\
\text { (promedios de 33 conos) }\end{array}$ & $\begin{array}{c}\text { Conos no polinizados } \\
\text { (promedios de 14 conos) }\end{array}$ & $\begin{array}{c}\text { Nivel de } \\
\text { significancia }\end{array}$ \\
\hline NPEF & $26.0( \pm 4.9)$ & $25.5( \pm 4.0)$ & $>0.05$ \\
NPSP & $51.9( \pm 9.7)$ & $51.0( \pm 8.1)$ & $>0.05$ \\
NPSD & $36.0( \pm 12.9)$ & $24.9( \pm 13.5)$ & 0.043 \\
NPSA & $16.1( \pm 9.7)$ & $26.4( \pm 12.3)$ & 0.024 \\
NPSLL & $18.7( \pm 14.1)$ & $6.7( \pm 10.6)$ & 0.033 \\
NPSV & $16.4( \pm 12.9)$ & $17.9( \pm 11.6)$ & $>0.05$ \\
\end{tabular}

* NPEF = número promedio de escamas fértiles; NPSP = número promedio de semillas potenciales; NPSD = número promedio de semillas desarrolladas; NPSA = número promedio de semillas abortadas; NPSLL = número promedio de semillas llenas; NPSV = número promedio de semillas vanas.

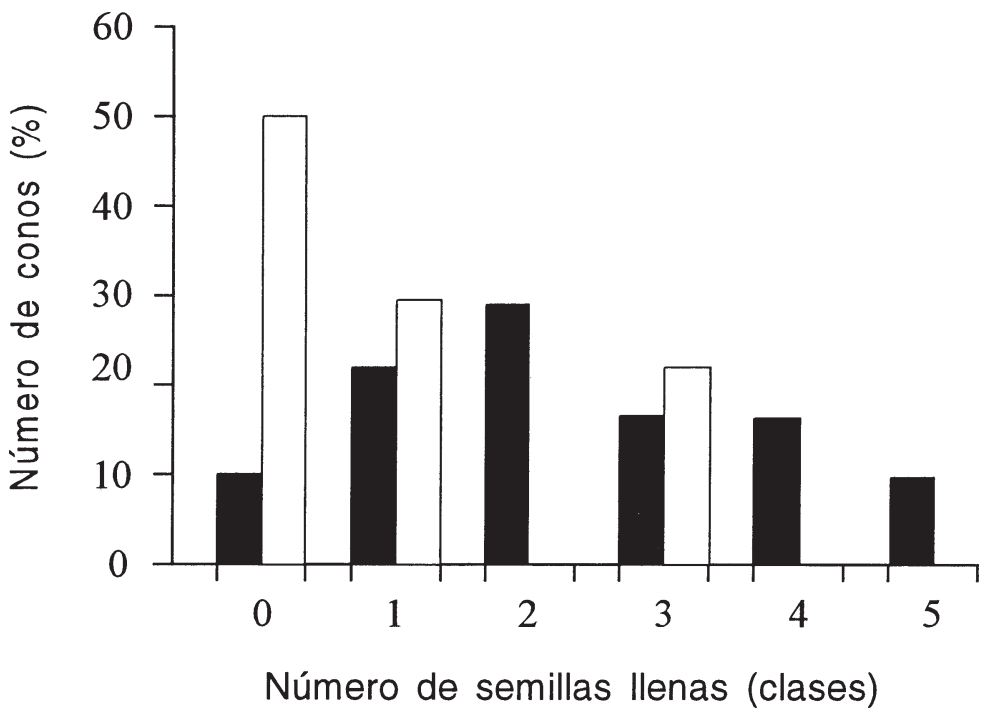

Fig. 2. Comparación entre la cantidad de conos con semillas llenas (clases) presentes en conos polinizados (barras negras) y en conos no polinizados (barras claras) de Pseudotsuga macrolepis. $(0=$ con semillas vanas; $1=$ con $1-10,2=11-20,3=21-30,4=31-40,5=41-50$ semillas llenas, respectivamente). 
Los conos protegidos contra insectos y los que no lo estuvieron mostraron diferencias estadísticamente significativas para las variables analizadas $(P<0.05)$, excepto los promedios de semillas vanas (NPSV) y abortadas (NPSA); destaca el menor número de las desarrolladas y llenas en los conos no protegidos (Cuadro 2). El de las desarrolladas (NSD) mostró correlación significativa $(P=0.0001)$ con el de escamas fértiles (NEF) $(r=0.65)$ (Cuadro 3). Aunado a esto, la longitud promedio de conos femeninos (LPCF) fue significativamente mayor en los protegidos que en los no protegidos $(P=0.005)$ (Cuadro 2).

Cuadro 2. Comparación entre el número de escamas y de semillas, así como el tamaño $(\mathrm{cm})$ en conos protegidos y no protegidos de Pseudotsuga macrolepis de Hidalgo.

\begin{tabular}{lccc}
\hline Variable $^{*}$ & $\begin{array}{c}\text { Conos protegidos } \\
\text { (promedios de 29 conos) }\end{array}$ & $\begin{array}{c}\text { Conos no protegidos } \\
\text { (promedios de 26 conos) }\end{array}$ & $\begin{array}{c}\text { Nivel de } \\
\text { significancia }\end{array}$ \\
\hline NPEF & $26.17( \pm 1.65)$ & $21.89( \pm 2.47)$ & 0.023 \\
NPSP & $52.33( \pm 3.29)$ & $43.77( \pm 4.95)$ & 0.023 \\
NPSD & $30.06( \pm 5.32)$ & $23.47( \pm 1.78)$ & 0.034 \\
NPSA & $22.38( \pm 4.05)$ & $20.49( \pm 3.72)$ & $>0.05$ \\
NPSLL & $13.24( \pm 3.69)$ & $7.39( \pm 4.66)$ & 0.015 \\
NPSV & $16.36( \pm 3.02)$ & $14.35( \pm 5.46)$ & $>0.05$ \\
LPCF & $5.82( \pm 0.23)$ & $4.76( \pm 0.56)$ & 0.005 \\
APCF & $1.54( \pm 0.17)$ & $1.83( \pm 0.10)$ & 0.005 \\
\hline
\end{tabular}

\footnotetext{
* NPEF = número promedio de escamas fértiles; NPSP = número promedio de semillas potenciales; NPSD = número promedio de semillas desarrolladas; NPSA = número promedio de semillas abortadas; NPSLL = número promedio de semillas llenas; NPSV = número promedio de semillas vanas; LPCF = longitud promedio de conos femeninos; APCF = ancho promedio de conos femeninos .
}

Tocante a las semillas vacías, de 81 conos estudiados se obtuvieron 1978 semillas desarrolladas, de las cuales $53.6 \%$ estuvieron vanas. El número promedio de estas últimas (NPSV) fue ligeramente mayor en conos no polinizados y protegidos que en los polinizados y sin protección, pero en ambos casos la diferencia no fue significativa (Cuadros 1 y 2 ). El número de semillas desarrolladas (NSD) mostró correlación positiva con el de las vanas (NPSV) ( $r=0.53, P=.0001)$ y el de éstas con el de las escamas fértiles (NEF) $(r=0.84$, $\mathrm{P}=.0001$ ) (Cuadro 3).

\section{DISCUSION}

El hallazgo de semillas desarrolladas (NPSD) y llenas (NPSLL) en conos que no fueron polinizados (Cuadro 1) nos llamó la atención. Existe la posibilidad que los conos que no fueron cubiertos con bolsas al inicio del experimento, para evitar la polinización, hayan estado contaminados con polen antes de polinizarlos artificialmente, pero esta situación no fue detectada. Sin embargo, según Allen (1942), los árboles de Pseudotsuga 
son capaces de desarrollar semillas por medio del mecanismo asexual de partenogénesis, que es el desarrollo apomíctico del óvulo y que incluye la producción de un embrión sin fecundación previa (Font Quer, 1979). La partenogénesis se ha señalado como un fenómeno de ocurrencia normal en Pseudotsuga menziesii (Mirb.) Franco (Allen, 1942). Según Larsen (1937), citado por Allen (1942), otros géneros de coníferas muestran partenogénesis (p. e. Larix), pero en Pseudotsuga dicho fenómeno puede ser obligado cuando los conos no son polinizados y hay condiciones adecuadas para su desarrollo. Se ha señalado que los conos partenogenéticos de Pseudotsuga suelen presentar pocas semillas llenas y muchas vanas (Allen, 1942). Esto concuerda con los resultados encontrados en el presente trabajo, en el cual los conos no polinizados mostraron mayor número de semillas vanas (NPSV) que llenas (NPSLL), así como una cantidad relativamente alta de abortadas (NPSA) (Cuadro 1).

Cuadro 3. Coeficientes de correlación entre las variables de número de escamas fértiles (NEF), número de semillas desarrolladas (NSD), Ilenas (NSLL) y vanas (NSV), por cono de Pseudotsuga macrolepis de Hidalgo (para todas las variables $n=73$ ).

\begin{tabular}{ccccc}
\hline & NEF & NSD & NSA & NSLL \\
NSD & .6536 & & & \\
& $(\mathrm{P}=.0001)$ & & & \\
NSA & .3605 & -.4690 & & \\
& $(\mathrm{P}=.002)$ & $(\mathrm{P}=.0001)$ & & \\
NSLL & .3128 & .6505 & -.4380 & \\
& $(\mathrm{P}=.007)$ & $(\mathrm{P}=.0001)$ & $(\mathrm{P}=.0001)$ & .2354 \\
NSV & .4812 & .5337 & -.0960 & $(\mathrm{P}>.05)$ \\
& $(\mathrm{P}=.0001)$ & $(\mathrm{P}=.0001)$ & $\mathrm{P}>.05$ & \\
\hline
\end{tabular}

La falta de diferencias significativas entre conos con polinización controlada y libre podría indicar un error en la selección del árbol donador de polen de la misma población, principalmente por ser ésta última poco numerosa y estar restringida a un área relativamente pequeña. No obstante, cabe la posibilidad que el polen depositado en las bolsas para cubrir los conos en el tratamiento de polinización controlada no haya sido suficiente. Según Ho (1980), el aislamiento de conos femeninos en bolsas con polen no garantiza la completa polinización de los mismos, ya que se requiere rociar varias veces con polen para lograr que cada óvulo reciba al menos un grano. Además, es común que los óvulos de conos de Pseudotsuga se hallen individualmente en diferentes etapas de madurez como resultado de un desarrollo asincrónico. El mismo autor encontró hasta $30 \%$ de óvulos sin polen germinado en conos aislados y polinizados una sola vez. Esto último pudo haber sucedido en los tratamientos de autopolinización y polinización libre, cuyos datos promedio de las variables analizadas tampoco mostraron diferencias significativas. 
Las diferencias significativas entre conos protegidos contra insectos y los que no lo estuvieron (Cuadro 2), así como las cantidades significativamente menores de semillas llenas en los no protegidos (Cuadro 1), parecen mostrar a los mismos como causantes de mortalidad de semillas de $P$. macrolepis en el área y durante el período de estudio. No obstante, de acuerdo con Hedlin (1964), debe considerarse que su efecto podría depender de la magnitud de la producción de conos, del grado de madurez de los mismos y, posiblemente, de la estación del año. Al respecto, Schenek et al. (1972) consignan que la producción de semillas de 70 árboles de $P$. menziesii en Idaho, E.U.A., en 1966, fue de unas 475000 , de las cuales cerca de $16 \%$ fueron destruidas por insectos al inicio de la estación de crecimiento. Nosotros encontramos que $45.7 \%$ de los 81 conos estudiados (que en conjunto podrían haber sido portadores de unas 1906 semillas) fueron dañados por insectos, desde $10 \%$ de su superficie hasta su totalidad. Con base en datos registrados por Contreras et al. (en prensa), suponemos que las lesiones observadas fueron causadas por Contarinia (Diptera, Cecidomyiidae), Megastigmus (Hymenoptera, Torymidae) y "trips" (Thysanoptera, Phlaeothripidae). Casi todos los conos dañados mostraron una mosquita (Contarinia sp.), la cual aparentemente fue la principal causante del daño. Lo encontrado por Contreras et al. (en prensa) apoya lo anterior; estos autores registraron, en 1989, que $69.4 \%$ de 1113 conos de Pseudotsuga macrolepis del área de estudio fueron dañados por diversos depredadores, pero casi $54 \%$ se debió a dicha mosquita. Hedlin (1964) registró la pérdida de 3-72 \% de la producción anual de semillas de Pseudotsuga por Contarinia, principalmente, en un período de 6 años. Dicho díptero oviposita varias veces en la base dorsal de las escamas de conillos femeninos; al eclosionar las larvas se alimentan de las escamas del cono, provocando un daño parcial pero importante para el desarrollo de semillas (Fig. 3).

De acuerdo con el Cuadro 2, parece ser que los insectos también provocaron la diferencia significativa $(P=0.03)$ en el número de semillas desarrolladas (NPSD) entre conos protegidos y sin protección. Esto posiblemente se debió a que las semillas que no se desarrollaron fueron depredadas en etapa de óvulo o abortaron. El aborto en $P$. macrolepis en el área estudiada parece ser un fenómeno importante. Yáñez (1991) menciona que en 1989 el aborto de óvulos de $P$. macrolepis en el área de estudio fue de $30 \%$ y que $41 \%$ de las semillas no completaron su desarrollo. Todavía falta investigar cuál es la razón por la que poco más de $1 / 3$ de las semillas producidas en una temporada no llegan a la madurez; Sorensen (1969) ha supuesto que el aborto en $P$. menziesii se debe al desarrollo incompleto de los óvulos durante la fase de crecimiento de los conos.

Las correlaciones significativas entre el número de semillas desarrolladas y el de escamas fértiles (Cuadro 3) y, tal vez, las diferencias entre longitud promedio de conos protegidos y sin protección (Cuadro 2), se pueden deber al ataque parcial de insectos a conos de Pseudotsuga, por lo cual estos últimos no pudieron alcanzar las dimensiones de los primeros. Por dicha razón, el número de escamas fértiles y, por consiguiente, el de semillas desarrolladas, se reduce (Ruth, 1980; Cibrián et al., 1986).

La correlación significativa entre el número de semillas vacías y el de las desarrolladas (Cuadro 3) indica que a mayor número potencial de semillas desarrolladas, mayor cantidad de semillas vacías; por consiguiente, estas últimas podrían deberse a causas inherentes a $P$. macrolepis, por ejemplo algún problema fisiológico o competencia entre óvulos en un cono, pero se carece de información al respecto. 
Los datos registrados en este trabajo sobre semillas vacías concuerdan con los de Yáñez (1991), quien encontró 55 \% de semillas vacías de conos de $P$. macrolepis en 1989. Schowalter et al. (1985) y Dombroski y Schowalter (1988) mencionan porcentajes semejantes a los antes dichos, pues consignan 44 y $58 \%$, respectivamente, para $P$. menziesii de Estados Unidos de América. Parece ser que la presencia de semillas vanas en Pseudotsuga es un problema importante y común, pero aún no hay claridad sobre las causas que la originan; algunos autores mencionan como posibles causantes la autopolinización, el aborto del embrión, así como la inviabilidad y escasez de polen (ElKassaby et al., 1984). No obstante, la falta de una diferencia significativa en el número

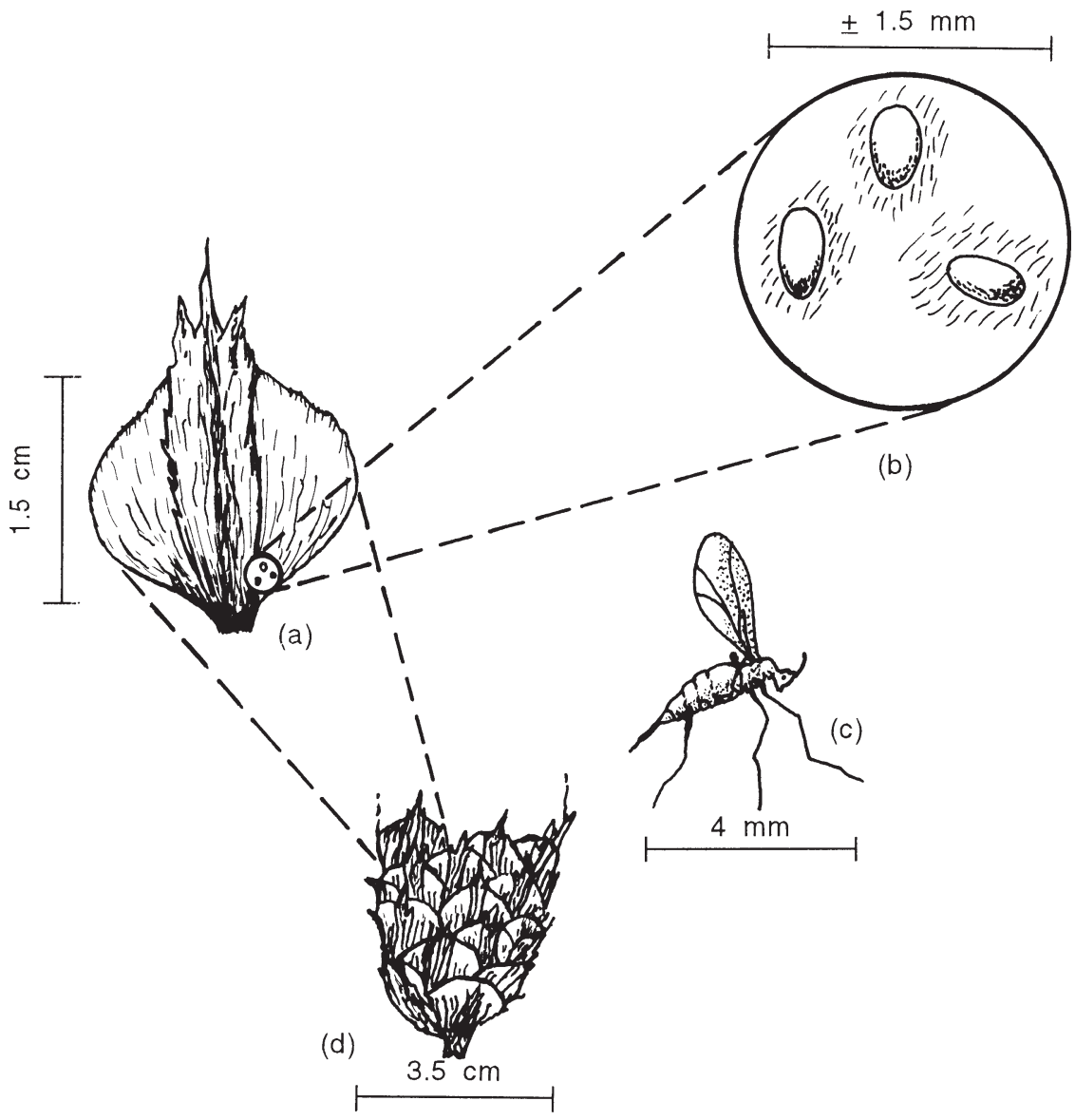

Fig. 3. Representación esquemática de Contarinia sp. (Diptera, Cecidomyiidae), uno de los principales factores bióticos causantes de la mortalidad de conos de Pseudotsuga macrolepis de Hidalgo (a. zona de la escama de un cono femenino de Pseudotsuga macrolepis donde ocurre la oviposición; b. huevecillos de Contarinia sp.; c. hembra madura de Contarinia sp.; d. mitad proximal de un cono femenino maduro de P. macrolepis). Adaptado de Hedlin et al., 1981. 
de semillas vacías entre conos autopolinizados y los polinizados libremente en este trabajo, podría indicar que la autopolinización no ocasiona la producción de semillas vanas en $P$. macrolepis en el área estudiada. Por otra parte, podrían considerarse otras razones, como la competencia por nutrimentos entre semillas y óvulos durante el desarrollo en el mismo cono, o también el hecho de que la producción de semillas vacías sea un fenómeno adaptativo (Willson, 1983), pues de esa manera se abate la competencia entre óvulos y semillas, pero estos aspectos no fueron estudiados.

Todavía se requiere investigar más sobre la producción de semillas de Pseudotsuga macrolepis; falta comprobar el fenómeno partenogénico, las causas de la presencia de semillas vacías, así como la dinámica de poblaciones de insectos relacionada con los rasgos fenológicos de $P$. macrolepis como un proceso de interacción planta-animal. La realización de estudios comparativos de estos aspectos en diferentes áreas de la distribución de la especie estudiada podrían arrojar alguna luz al respecto.

\section{CONCLUSIONES}

La polinización insuficiente y el ataque de insectos son factores que parecen estar involucrados en la producción de semillas de Pseudotsuga macrolepis en la Sierra de Pachuca, Hgo., pero falta precisar la importancia relativa de ambas. En el primer caso, la baja densidad de la población de dicha especie en el área estudiada tal vez evita el suministro de polen suficiente y de manera reincidente, pues esto es requerido ante la posible maduración asincrónica de óvulos en conos de Pseudotsuga. Debido al aborto de semillas potenciales y a la presencia de las vanas, sólo cerca de 8 semillas por cono quedan disponibles para la regeneración.

Aunque posiblemente hay desarrollo de semillas por partenogénesis, se puede considerar la necesidad de repoblación artificial de $P$. macrolepis en el área, a fin de recuperar la abundancia de esta especie. Es posible que el carácter pequeño de la población también contribuya en el efecto de los insectos fitófagos en la producción de conos y semillas de Pseudotsuga en el área, pero falta documentar su importancia como consumidores con base en información de varios años.

\section{AGRADECIMIENTOS}

El presente trabajo fue realizado gracias al financiamiento otorgado por la Universidad Autónoma Chapingo (proyecto 8901011). Agradecemos a los ejidatarios del Ejido El Cerezo, Pachuca, Hgo., las facilidades para este estudio; al M. C. Victor Manuel Cetina Alcalá, del Programa Forestal del Colegio de Postgraduados, y en especial al Dr. Jaime Flores Lara, de la Facultad de Ciencias Forestales de la Universidad Autónoma de Nuevo León, por sus atinados comentarios y críticas al escrito final. Asimismo, a los revisores anónimos de Acta Botánica Mexicana por sus sugerencias para la presentación escrita de este trabajo, nuestro agradecimiento. 


\section{LITERATURA CITADA}

Allen, G. S. 1942. Parthenocarpy, parthenogenesis, and self-sterility of douglas-fir. J. Forestry 40: 642644.

Anónimo. 1982. Carta topográfica F-14-D81. Esc. 1:50 000. Instituto Nacional de Estadística, Geografía e Informática. México, D.F.

Bramlett, D. L., E. W. Belcher, Jr., G. L. Debarr, G. D. Hertel, R. P. Karrfalt, C. W. Lants, T. Miller, K. D. Ware, y H. O. Yates III. 1977. Cone analysis of southern pines: a guidebook. U. S. Department of Agriculture, Forest Service. Gen. Tech. Rep. SE-13, 28 pp.

Cibrián T., D., D. H. Ebel, H. O. Yates III, y J. T. Méndez M. 1986. Insectos de conos y semillas de las coníferas de México. Cone and seed insects of the Mexican conifers. Universidad Autónoma Chapingo - Secretaría de Agricultura y Recursos Hidráulicos, México - United States Department of Agriculture, Forest Service. $110 \mathrm{pp}$.

Contreras A., R., R. Campos B., J. T. Méndez M. y F. Zavala Ch. (en prensa). Factores de mortalidad de conos de Pseudotsuga macrolepis Flous. In: Pineda, M. del C. (ed.). Memorias del V Simposio Nacional sobre Parasitología Forestal. Cd. Juárez, Chih. 7 pp.

Dombroski, S. A. y T. D. Schowalter. 1988. Inventary monitoring for estimating impact of insects on seed production in a douglas-fir seed orchard in Western Oregon. J. Econ. Entomol. 81(1): 281-285.

El-Kassaby, Y. A., A. M. K. Fashler y O. Szilklal. 1984. Reproductive phenology and its impact on genetically improved seed production in a douglas-fir seed orchard. Silvae Genet. 33(4-5): 120125.

Font Quer, P. 1979. Diccionario de botánica. Editorial Labor, S. A. Barcelona. 1244 pp.

García, E. 1973. Modificaciones al sistema de clasificación climática de Köppen (para adaptarlo a las condicones de la República Mexicana). Instituto de Geografía, Universidad Nacional Autónoma de México. México, D.F. 243 pp.

Hedlin, A. F. 1964. Results of a six-year plot study on douglas-fir cone insect population fluctuations. For. Sci. 10(1): 124-128.

Hedlin, A. F., H. O. Yates III, D. Cibrián T., B. H. Ebel, T. W. Koerber y E. P. Merkel. 1981. Cone and seed of North American conifers. Canadian Forestry Service, United States Forest Service, Secretaría de Agricultura y Recursos Hidráulicos, México, D.F. 122 pp.

Ho, R. H. 1980. Pollination mechanism and seed production potential in douglas-fir. For. Sci. 26(4): 522528.

Hutchinson, J. G. y D. L. Bramlett. 1964. Forest damage to short leaf pine flowers. J. Forestry 62: 343.

Medina, J. M. y J. Rzedowski. 1981. Sierra de Pachuca. In: Sociedad Botánica de México, A. C. (ed.). Guías botánicas de México. Sociedad Botánica de México, A. C. México, D. F. Vol. IV, pp. 119.

Owens, J. N. 1986. Cone and seed biology. In: Shearer, R. C. (comp.). Proceedings of the conifer tree in the Inland Mountain West Symposium (1985). U. S. Department of Agriculture, Forest Service; Intermountain Research Station. Gen. Tech. Rep. Int. 203. pp. 14-31.

Rehfeldt, G. E., A. R. Stage y R. T. Bingham. 1971. Strobili development in western white pine: periodicity, prediction, and association with weather. For. Sci. 17(4): 454-561.

Ruth, D. S. 1980. A guide to insects pests in douglas-fir seed orchards. Forest Service, Pacific Forest and Environment Research Center. Victoria, Canada. Report BC-X-204. 19 pp.

Scheneck, J. A., D. O. Everson, y J. R. Gosz. 1972. Sampling cone production in douglas-fir stands for insects populations studies. Forest, Wildlife and Range Experimental Station. University of Idaho, College of Forestry, Wildlife and Range Sciences. Station Paper No. 10. 10 pp.

Schowalter, T. D., M. I. Haverty, y T. W. Koerber. 1985. Cone and seed insects in douglas-fir, Pseudotsuga menziesii (Mirb.) Franco, seed orchards in the western United States: distribution and relative impact. Can. Ent. 117: 1223-1230.

Sorensen, F. 1969. Embryonic genetic load in coastal douglas-fir, Pseudotsuga menziesii var. menziesii. Am. Nat. 103(932): 389-398. 
Zavala y Méndez: Factores que Afectan la Producción de Semillas en Pseudotsuga macrolepis

Steel, R. G. D. y J. H. Torrie. 1985. Biostadística: principios y procedimientos. (2a. ed.) McGraw-Hill. Bogotá. $622 \mathrm{pp}$.

Willson, M. F. 1983. Plant reproductive ecology. John Wiley \& Sons. Nueva York. 282 pp.

Yáñez E., L. 1991. Análisis de la producción de semillas de Pseudotsuga macrolepis Flous en una población natural de la Sierra de Pachuca, Hidalgo. Tesis Profesional. División de Ciencias Forestales, Universidad Autónoma Chapingo. Chapingo, México. 116 pp. 